\title{
Multimodality Imaging Diagnosis of Multiple Ventricular Thrombosis and Massive Stroke after Gemcitabine and Cisplatin Chemotherapy for Urothelial Cancer
}

\author{
Pier Paolo Bassareo ${ }^{1,2}$, Daniele Cocco², Christian Cadeddu², Giuseppe Mercuro \\ ${ }^{1}$ Department of Cardiology, University College of Dublin, Mater Misericordiae University Hospital, Dublin, Republic of Ireland, \\ 2Department of Medical Sciences and Public Health, University of Cagliari, Monserrato, Italy
}

\section{Abstract}

Cancer and chemotherapy are known to be risk factors for developing coagulative disorders, venous thrombosis, adverse cardiovascular events, and cardiotoxicity. Combined modality gemcitabine-cisplatin chemotherapy is often administered to treat a few solid tumors. We report the unusual case of a man suffering from urothelial cancer and admitted for chemotherapy, who developed an ischemic stroke after the last chemotherapeutical cycle. During his hospital stay, at echocardiographic examination, left ventricular transient hypokinesia and two intraventricular thrombi were detected, without evidence of acute coronary syndrome. Multimodality imaging approach (i.e., transthoracic echo, transoesophageal echo, computed tomography, and cardiac magnetic resonance imaging) played a pivotal role for a clear diagnosis and prompt decision-making. This is the first report of an intraventricular-related arterial thromboembolic event in a patient treated with the combination gemcitabine-cisplatin.

Keywords: Cancer, cardiac magnetic resonance imaging, chemotherapy, computed tomography, echocardiography, thromboembolism

\section{INTRODUCTION}

Cancer and chemotherapy are known to be risk factors for developing coagulative disorders, thrombosis, adverse cardiovascular events, and cardiotoxicity. ${ }^{[1,2]}$ Combined modality gemcitabine-cisplatin chemotherapy is often administered to treat a few solid tumors, including non-small cell lung cancer, esophageal, gastric, testicular, and urothelial neoplasms. ${ }^{[3]}$

Among the chemotherapy-induced side effects, venous thromboembolism is significantly more frequent than arterial thrombotic disorders. The latter indeed may be asymptomatic, and therefore, potentially underdiagnosed, and conversely, sometimes may result in myocardial infarction, stroke, or peripheral embolism. ${ }^{[3]}$

Although not yet fully understood, several mechanisms appear to be involved, including elevated von Willebrand factor levels, endothelial damage, hypomagnesemia, and microangiopathy. ${ }^{[3]}$

\begin{tabular}{|l|l|}
\hline \multicolumn{2}{|c|}{ Access this article online } \\
\hline Quick Response Code: & Website: \\
& www.jcecho.org \\
\cline { 2 - 2 } & \\
\hline
\end{tabular}

\section{Case Report}

We report the unusual case of a 60 -year-old male suffering from urothelial cancer and admitted to the oncology department for gemcitabine-cisplatin chemotherapy repeating cycles. Four months before that, the patient had been surgically treated for urothelial cancer (Stage pT2aG3) by means of transurethral resection of the bladder, and a double-J catheter had been inserted. Because of the risk of developing cardiotoxicity, serial echocardiograms displaying normal findings were performed before starting chemotherapy and during cycles.

During his hospital stay, the patient developed an ischemic stroke. Six consecutive chemotherapy cycles had been administered (cisplatin $75 \mathrm{mg} / \mathrm{m}^{2}$ and gemcitabine

Address for correspondence: Prof. Pier Paolo Bassareo, University College of Dublin, Mater Misericordiae University Hospital, Eccles St, Inns Quay, Dublin 7, D07 R2WY, Dublin, Republic of Ireland. E-mail: piercard@inwind.it

This is an open access journal, and articles are distributed under the terms of the Creative Commons Attribution-NonCommercial-ShareAlike 4.0 License, which allows others to remix, tweak, and build upon the work non-commercially, as long as appropriate credit is given and the new creations are licensed under the identical terms.

For reprints contact: reprints@medknow.com

How to cite this article: Bassareo PP, Cocco D, Cadeddu C, Mercuro G. Multimodality imaging diagnosis of multiple ventricular thrombosis and massive stroke after gemcitabine and cisplatin chemotherapy for urothelial cancer. J Cardiovasc Echography 2019;29:71-4. 
$1000 \mathrm{mg} / \mathrm{m}^{2}$ ) before that. The last chemotherapy cycle had been administered 3 days before the stroke. Based on these premises, he was transferred to the neurology department due to difficulty in speaking, severe and pulsatile right headache, confusion, and left-hand side hemianopsia. An urgent computed tomography (CT) brain scan without contrast showed a wide hypodense ischemic lesion at parietal and occipital right brain regions with compression of the homolateral ventricle and without hemorrhagic infarct [Figure 1].

Blood tests displayed mild hypomagnesemia $(0.7 \mathrm{mmol} / \mathrm{l})$. Thrombocytopenia (53,000 cells/dl), likely due to the recent chemotherapy, was also detected.

The patient had no classic risk factors for coronary artery disease. His blood pressure was $125 / 80 \mathrm{mmHg}$ and his physical examination was unremarkable. Basal electrocardiogram (ECG) showed sinus rhythm with normal morphology. Cardiac enzymes and troponins were within the normal range. Aortic atherosclerotic plaques had been excluded by a previous radiological examination. Echo scan of the carotid vessels showed nonsignificant atherosclerosis at his left internal carotid artery.

Due to the considerable extension of the ischemic brain lesion, a cardioembolic origin was hypothesized, and the patient was examined by means of transthoracic echocardiography. Two thrombi were detected in the left ventricle: the first $(2.3 \mathrm{~cm} \times 0.8 \mathrm{~cm})$ was located in the heart apex, whereas the second $(2.5 \mathrm{~cm} \times 1.1 \mathrm{~cm})$ was floating, ball-shaped, and stitched to the interventricular septum by means of a short, thin peduncle [Figure 2]. Transient mild apical, mid-septal, and mid-anterior wall hypokinesia with concomitant preserved global ejection fraction (EF 59\%) was also observed. These data were confirmed at transoesophageal echocardiography as well [Figure 3]. At CT thoracic scan, only the cardiac apical thrombus was clearly identified [Figure 4]. ECG was unchanged.

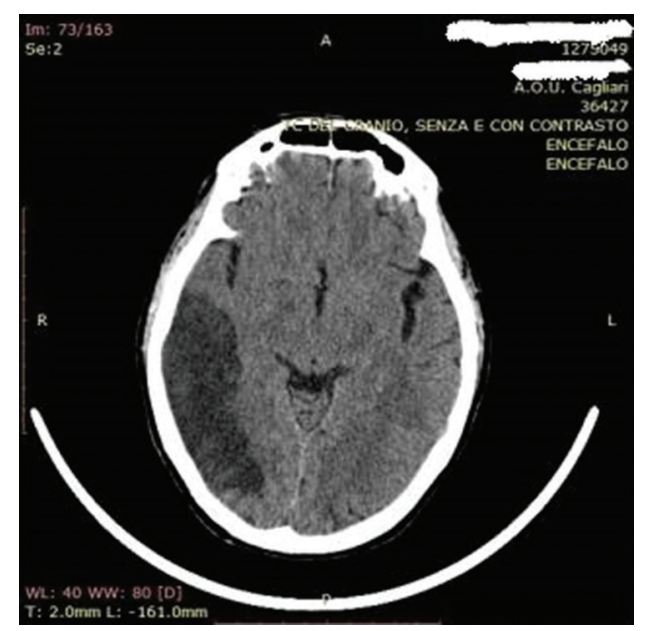

Figure 1: Computed tomography brain scan shows an extensive ischemic stroke at the occipital and parietal right brain regions
Following a diagnosis of cardioembolic stroke, the patient was transferred to the cardiology department and treated with fondaparinux $(7.5 \mathrm{mg} /$ day). Chemotherapy had already been finished with the sixth gemcitabine-cisplatin cycle, as usual in oncology protocols. Ischemia-inducible tests were not prescribed due to the patient's high embolic risk. However, his coronary arteries were patent at angiography. On the contrary, coagulation screening showed an increase in both von Willebrand factor Ag levels $(189 \%$, reference range: $52 \%-143 \%$ ) and von Willebrand factor activity ( $218 \%$, reference range: $52 \%-136 \%$ ). During hospitalization, the patient did not make complaints for cardiac symptoms (chest pain, dyspnea, palpitations, and syncope). At subsequent transthoracic echocardiographic controls, hypokinesia and apical thrombus disappeared after 21 and 25 days, respectively, whereas septal thrombus reduction occurred at a slower rate. Forty-four days after admission, a contrast-enhanced cardiac magnetic resonance (1.5 scanner, Signa Hdx; GE Healthcare, Milan, Italy) revealed a significant reduction in septal thrombus dimensions (about $0.9 \mathrm{~cm} \times 0.6 \mathrm{~cm})$ and absent late gadolinium enhancement. Fast imaging employing steady-state acquisition sequences allowed a clear delineation between the residual thrombus and intraventricular blood (black pool). Global EF was preserved (68\%) [Figure 5a and b].

\section{Discussion}

Cancer patients receiving chemotherapy are at risk of developing thromboembolic adverse events, disorders of venous origin being decidedly more frequent than the arterial.

Cisplatin, a platinum-based chemotherapy drug, is associated with an increased risk of thromboembolic events although this association is merely based on case reports and retrospective studies and not confirmed in meta-analyses.$^{[3]}$ Cisplatin-induced thromboembolic events tend to occur early in the course of treatment, with about half occurring over the first two courses

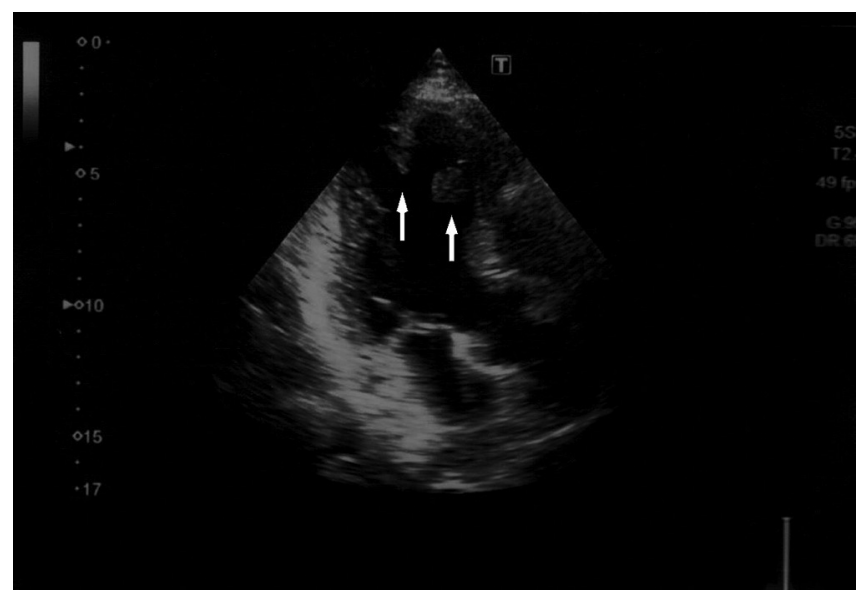

Figure 2: Transthoracic echocardiogram: Apical three-chamber view shows two thrombi within the left ventricle: One stitched to the apex and another to the interventricular septum (white arrows) 
Bassareo, et al.: Imaging in cardiotoxicity

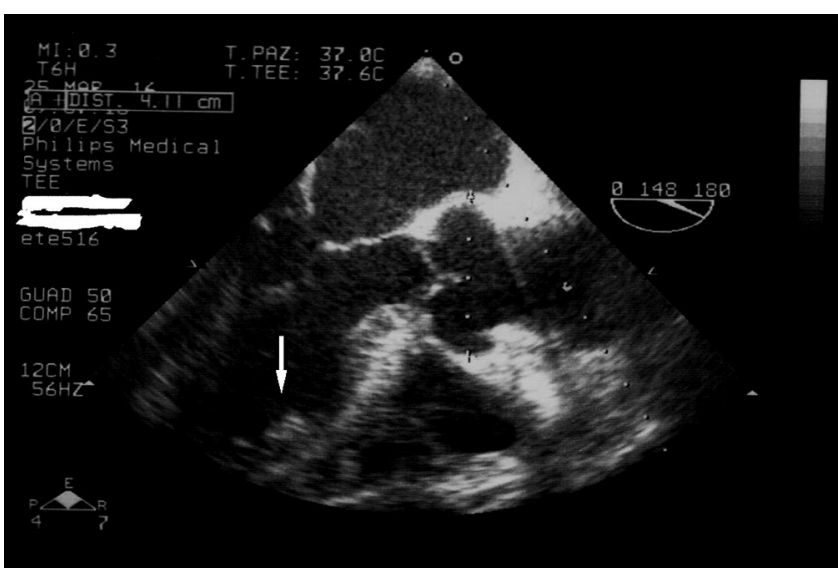

Figure 3: Transoesophageal echocardiogram: The interventricular thrombus is shown (white arrow)

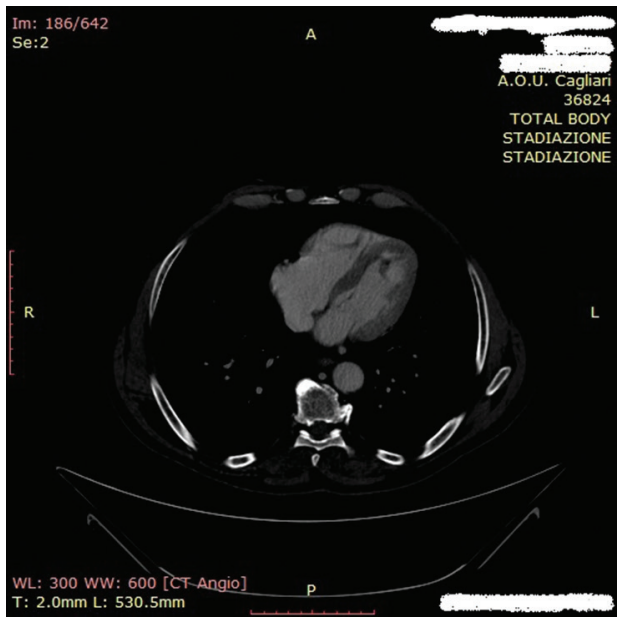

Figure 4: Computed tomography scan displays the apical thrombus. Otherwise, no other abnormal findings were detected
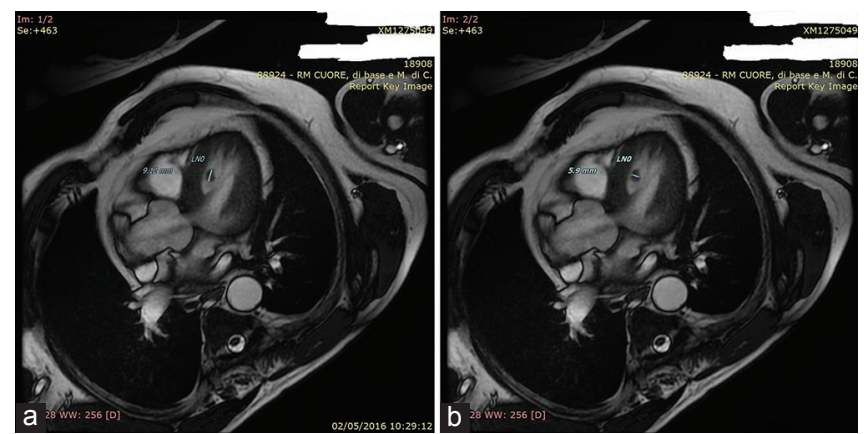

Figure 5: ( $a$ and $b)$ Cardiac magnetic resonance imaging: Four-chamber view displays a residual ventricular thrombus. Steady-state free precession allows an excellent contrast between myocardium and blood within the heart (blood pool)

of chemotherapy. The mechanism by which cisplatin leads to vascular events is presently unknown, although endothelial damage would appear to play a major role. Other suggested hypotheses are cisplatin-induced elevation of plasma levels of von Willebrand factor and drug-induced reduction of left ventricular function. With regard to the latter, several cases of
Takotsubo-like transient myocardial stunning were described. ${ }^{[4]}$ Furthermore, cisplatin may cause hypomagnesemia, which in turn is associated with vasospasm and consequent hemostasis and thrombosis. ${ }^{[5]}$

On the other hand, gemcitabine, an analog of the antimetabolite ara- $\mathrm{C}$, is associated with an increased risk of frequently venous and rarely arterial thromboembolic events. Again, also these harmful side effects were reported more frequently in case reports and case series but not confirmed in the meta-analyses. ${ }^{[6]}$ Regarding the underlying mechanism, gemcitabine may lead to thrombotic microangiopathy, a rare and potentially fatal complication. Microangiopathy is a microvascular occlusive disorder characterized by predominantly platelet thrombi in the systemic circulation. ${ }^{[7]}$ This type of toxicity appears to be more frequent when using gemcitabine-platinum doublets than with gemcitabine alone, the arterial events being anecdotal compared with the venous. ${ }^{[3,8]}$

Finally, a hypercoagulable state of malignancy per se is capable of inducing the formation of thrombi. A high incidence of thromboembolic events was recently described in patients affected by urothelial cancer who were treated with cisplatin and without other risk factors for clots formation. ${ }^{[9]}$

All the above mechanisms may be responsible, at least in part, for the onset of the two thrombi in the heart of our patient.

To the best of our knowledge, only one case of intraventricular thrombosis was previously reported in the literature, related to a patient with esophageal cancer, treated with cisplatin alone, and with concomitant persistent reduced left ventricular ejection. ${ }^{[10]}$ This is the first report of an intraventricular-related arterial thromboembolic event in a patient treated with the combination gemcitabine-cisplatin.

In our case report, left ventricular dysfunction was transient, global EF was normal, and there was no evidence of coronary artery disease. Chemotherapy-induced thrombosis, therefore, seems to be the most likely cause underlying the intraventricular appearance of the two thrombi. Further scientific evidence should be provided to fully understand the mechanisms underlying the onset of chemotherapy-induced arterial thrombosis, which frequently becomes symptomatic only at the time of a dramatic adverse embolic event, as in the case of our patient.

A combined multimodality imaging approach, including transthoracic echo, transoesophageal echo, computed tomography, and cardiac magnetic resonance imaging, played a pivotal role for a clear diagnosis and prompt decision-making. ${ }^{[1]}$

\section{Declaration of patient consent}

The authors certify that they have obtained all appropriate patient consent forms. In the form, the patient has given his consent for his images and other clinical information to be reported in the journal. The patient understands that name and initials will not be published and due efforts will be made to conceal identity, but anonymity cannot be guaranteed. 
Bassareo, et al.: Imaging in cardiotoxicity

\section{Financial support and sponsorship}

Nil.

\section{Conflicts of interest}

There are no conflicts of interest.

\section{RefERENCES}

1. Cadeddu Dessalvi C, Deidda M, Mele D, Bassareo PP, Esposito R, Santoro C, et al. Chemotherapy-induced cardiotoxicity: New insights into mechanisms, monitoring, and prevention. J Cardiovasc Med (Hagerstown) 2018;19:315-23.

2. Ciliberti G, Carotti L, Russo N, Gioia MI, Caroli G, Guerra F, et al. An unusual left atrial mass in a woman with active breast cancer and recent cardiothoracic surgery. J Cardiovasc Echogr 2018;28:51-3.

3. Proverbs-Singh T, Chiu SK, Liu Z, Seng S, Sonpavde G, Choueiri TK, et al. Arterial thromboembolism in cancer patients treated with cisplatin: A systematic review and meta-analysis. J Natl Cancer Inst 2012;104:1837-40.

4. Ono R, Falcão LM. Takotsubo cardiomyopathy systematic review: Pathophysiologic process, clinical presentation and diagnostic approach to takotsubo cardiomyopathy. Int J Cardiol 2016;209:196-205.
5. Fernandes DD, Louzada ML, Souza CA, Matzinger F. Acute aortic thrombosis in patients receiving cisplatin-based chemotherapy. Curr Oncol 2011;18:e97-100.

6. Qi WX, Lin F, Sun YJ, Tang LN, Shen Z, Yao Y. Risk of venous and arterial thromboembolic events in cancer patients treated with gemcitabine: A systematic review and meta-analysis. $\mathrm{Br} \mathrm{J}$ Clin Pharmacol 2013;76:338-47.

7. Izzedine $\mathrm{H}$, Isnard-Bagnis $\mathrm{C}$, Launay-Vacher V, Mercadal L, Tostivint I, Rixe $\mathrm{O}$, et al. Gemcitabine-induced thrombotic microangiopathy: A systematic review. Nephrol Dial Transplant 2006;21:3038-45.

8. Ak G, Metintas S, Akarsu M, Metintas M. The effectiveness and safety of platinum-based pemetrexed and platinum-based gemcitabine treatment in patients with malignant pleural mesothelioma. BMC Cancer 2015;15:510.

9. Tully CM, Apolo AB, Zabor EC, Regazzi AM, Ostrovnaya I, Furberg HF, et al. The high incidence of vascular thromboembolic events in patients with metastatic or unresectable urothelial cancer treated with platinum chemotherapy agents. Cancer 2016;122:712-21.

10. Morlese JF, Jeswani T, Beal I, Wylie P, Bell J. Acute ventricular and aortic thrombosis post chemotherapy. Br J Radiol 2007;80:e75-7.

11. Bencini C, Corrado G, Borghi C, Carugo S. Multimodality imaging of left ventricular clefts in an asymptomatic teenager. J Cardiovasc Echogr 2018;28:182-4. 